\title{
An adaptive Hybridizable Discontinuous Galerkin approach for cardiac electrophysiology
}

\author{
J. M. Hoermann ${ }^{1}$, C. Bertoglio ${ }^{2,3}$, M. Kronbichler ${ }^{1 *}$, M. R. Pfaller ${ }^{1}$, R. Chabiniok $^{4,5,6}$ \\ and W. A. Wall ${ }^{1}$ \\ ${ }^{1}$ Institute for Computational Mechanics, Technical University Munich Boltzmannstr. 15, 85748 Garching b. München, \\ Germany \\ ${ }^{2}$ Center for Mathematical Modeling, Universidad de Chile, Beaucheff 851, 8370456 Santiago, Chile \\ ${ }^{3}$ Johann Bernoulli Institute, University of Groningen, Nijenborgh 9, 9747 HZ Groningen, Netherlands \\ ${ }^{4}$ Inria, Paris-Saclay University, Palaiseau, France \\ ${ }^{5}$ LMS, Ecole Polytechnique, CNRS, Paris-Saclay University, Palaiseau, France \\ ${ }^{6}$ School of Biomedical Engineering \& Imaging Sciences (BMEIS), St Thomas' Hospital, King's College London, UK
}

\section{SUMMARY}

Cardiac electrophysiology simulations are numerically challenging due to the propagation of a steep electrochemical wave front and thus require discretizations with small mesh sizes to obtain accurate results. In this work, we present an approach based on the Hybridizable Discontinuous Galerkin method (HDG), which allows an efficient implementation of high-order discretizations into a computational framework. In particular using the advantage of the discontinuous function space, we present an efficient p-adaptive strategy for accurately tracking the wave front. HDG allows to reduce the overall degrees of freedom in the final linear system to those only on the element interfaces. Additionally, we propose a rule for a suitable integration accuracy for the ionic current term depending on the polynomial order and the cell model to handle high-order polynomials. Our results show that for the same number of degrees of freedom coarse high-order elements provide more accurate results than fine low-order elements. Introducing $\mathrm{p}$ adaptivity further reduces computational costs while maintaining accuracy by restricting the use of highorder elements to resolve the wave front. For a patient-specific simulation of a cardiac cycle p-adaptivity reduces the average number of degrees of freedom by $95 \%$ compared to the non-adaptive model. In addition to reducing computational costs, using coarse meshes with our p-adaptive high-order HDG method also simplifies practical aspects of mesh generation and postprocessing. Copyright @ 2017 John Wiley \& Sons, Ltd.

Received ...

KEY WORDS: cardiac electrophysiology, finite element method, Hybridizable Discontinuous Galerkin

\section{INTRODUCTION}

Biophysical and computational modeling of the heart has been proposed and actively pursued as a tool for accelerating cardiovascular research and translation to clinic to personalize the care is very promising [1]. One of the challenges modelers have to face is the high cost of the computations, in particular when moving towards whole heart modeling and coupling different physics and scales. To develop methods that reduce the computing time while keeping numerical accuracy is essential for speeding-up fundamental research but most importantly the translation of modeling into clinical practice.

\footnotetext{
${ }^{*}$ Correspondence to: Institute for Computational Mechanics, Technical University Munich, Boltzmannstr. 15, 85748 Garching b. München, Germany. E-mail: kronbichler@lnm.mw.tum.de 
Cardiac electrophysiology simulations are classically based on monodomain or bidomain reaction-diffusion equations for the transmembrane electrical potential, which are coupled to a model for the gating dynamics of the ionic channels on the cell membrane. Due to the fast upstroke of the action potential, which is caused by voltage-dependent sodium channels, a numerically robust calculation of the propagation of the wave across the tissue is well known to be computationally challenging. This rapid increase of the transmembrane potential in one cell over a few milliseconds results also in a steep wave front in space [2], requiring high resolution temporal and spatial discretizations [3].

Galerkin methods are popular in cardiac electrophysiology due to their simple applicability to complex geometries and higher-order discretizations [4, 5, 6, 7, 8, 9, 10, 11, 12]. Attempts have been made to improve the performance of the numerical method by using h-adaptivity in space [13, 14, 15], adaptivity in time [6, 16, 17] and in both space and time [18, 19, 20, 21, 22]. Adaptivity in space based on low order elements requires remeshing during the calculation, which involves a considerable computational effort. Furthermore, this approach has to couple the numerical solver with a mesh generator. Therefore, studies about efficient simulations with high-order elements and high-order p-adaptive elements were performed for continuous Galerkin (CG) in [23, 24]. Highorder discretizations have been shown to be more efficient than low-order ones for a variety of contexts, i.e., they achieve better accuracy with fewer degrees of freedom and less computational cost [24, 25]. However, the adaption of the polynomial degree in CG faces additional challenges, e.g. for preserving continuity between the elements [26].

To the authors' best knowledge, discontinuous Galerkin (DG) approaches have not received any attention yet for the electrophysiology problem. The discontinuous setting allows a straightforward implementation of global or local high-order discretizations and/or local mesh refinement.

The hybridizable discontinuous Galerkin (HDG) method [27] is a special case of the DG family of methods that allows a reduction of the degrees of freedom that appear in the final linear system by static condensation on the element to those defined on the faces between the elements. The goal of this work is to present a suitable HDG formulation for cardiac electrophysiology, study the numerical performance of HDG in this context and to compare it against standard CG approaches.

The remainder of the paper is organized as follows. In Section 2 we summarize the electrophysiology model problem and recall the CG and HDG discretization approaches. We then discuss the strategies used for the calculation of the ionic current term with focus on high polynomial degrees of the function spaces. In Section 3 we detail the setups of the numerical experiments and the cell model we use, and exemplify the ionic current integration rule for a specific cell model. We also determine a practical choice for the stabilization parameter in HDG. The numerical results comprise an academic geometry for benchmarking purposes and a simulation on a patient-specific heart, where both CG and (p-adaptive) HDG methods are assessed.

\section{METHODS}

\subsection{Electrophysiology equations}

The classical monodomain model is given in the domain $\Omega \subset \mathbb{R}^{3}$ in the following way: Find the transmembrane potential $u: \Omega \times t \rightarrow \mathbb{R}$ and the gating variables $\mathbf{w}: \Omega \times t \rightarrow \mathbb{R}^{m}$ such that

$$
\left\{\begin{aligned}
\chi\left(C_{m} \partial_{t} u-I_{i o n}(u, \mathbf{w})\right) & =\nabla \cdot(\mathbf{D} \nabla u) & & \text { in } \Omega \times(0, T], \\
\partial_{t} \mathbf{w}-\mathbf{g}(u, \mathbf{w}) & =0 & & \text { in } \Omega \times(0, T], \\
\mathbf{n} \cdot(\mathbf{D} \nabla u) & =0 & & \text { on } \partial \Omega \times(0, T], \\
u(x, 0) & =u_{0}(x) & & \text { in } \Omega, \\
\mathbf{w}(x, 0) & =\mathbf{w}_{0}(x) & & \text { in } \Omega .
\end{aligned}\right.
$$

The physical (given) constants are: the ratio of membrane area per tissue volume $\chi$, the local membrane capacitance $C_{m}$, the electrical conductivity tensor $\mathbf{D}$, the initial conditions $u_{0}$ and $w_{0}$, and the outward pointing unit normal vector $\mathbf{n}$ to $\partial \Omega$. The given non-linear functions $I_{\text {ion }}: \mathbb{R}^{m+1} \rightarrow$ 
$\mathbb{R}$ and $\mathbf{g}: \mathbb{R}^{m+1} \rightarrow \mathbb{R}^{m}$ describe the total ionic current flow through the membrane of the myocyte and the kinetics of the gating variables as defined by the cell model, respectively.

\subsection{Time semidiscretization}

For the sake of clarity of the presentation, we first introduce the time marching scheme used. It is important to remark that the choice is independent on the spatial discretization. We adopt a semiimplicit backward Euler approach: the diffusion term is discretized implicitly in time, while the reaction term is evaluated explicitely [28, 29]. Assuming uniform time intervals $t_{0}, t_{1}, \ldots$ with $d t=t_{n}-t_{n-1} \forall n>0$, the time semidiscrete problem reads: Given $u^{0}, \mathbf{w}^{0}$, find the transmembrane potential $u^{n}: \Omega \rightarrow \mathbb{R}$ and the gating variables $\mathbf{w}^{n}: \Omega \rightarrow \mathbb{R}^{m}$ for $n>0$ such that

$$
\left\{\begin{aligned}
\chi\left(C_{m} \frac{u^{n}-u^{n-1}}{d t}-I_{i o n}\left(u^{n-1}, \mathbf{w}^{n-1}\right)\right) & =\nabla \cdot\left(\mathbf{D} \nabla u^{n}\right) & & \text { in } \Omega, \\
\frac{\mathbf{w}^{n}-\mathbf{w}^{n-1}}{d t}-\mathbf{g}\left(u^{n}, \mathbf{w}^{n}\right) & =0 & & \text { in } \Omega, \\
\mathbf{n} \cdot\left(\mathbf{D} \nabla u^{n}\right) & =0 & & \text { on } \partial \Omega
\end{aligned}\right.
$$

Note that this scheme allows important gains in computational efficiency: the problem for $u^{n}$ is linear and hence system matrices and preconditioners are assembled only once at the beginning of the computation. CFL-like restrictions on the time stepping, which arise from spatial derivatives and are thus mesh-dependent, are avoided due to the implicit treatment of the diffusion terms. The latter can be of particular interest when dealing with spatial adaptivity as it is done in this work. Finally, note that since the cell model used in this work is linear in the gating variables, and hence only linear problems have to be solved in the implicit parts.

\subsection{Continuous Galerkin approximation}

In this section we describe the spatial discretization of Equation (2) by a Continuous Galerkin approximation.

Let us assume a domain $\Omega$ and a triangulation $\mathcal{T}_{h}$ of this domain with elements of characteristic size $h$. We will denote each of these elements as $K$. Let us recall the standard function spaces $V_{h}^{p}$ of piecewise continuous polynomials of degree $p$ :

$$
V_{h}^{p}=\left\{v \in H^{1}(\Omega):\left.v\right|_{K} \in \mathcal{P}^{p}(K) \text { for element } K \in \mathcal{T}_{h}\right\}
$$

where $H^{1}(\Omega)$ denotes the Sobolev space $W^{1,2}(\Omega)$ and $\mathcal{P}^{p}(K)$ the set of polynomials of maximal degree $p$ on a domain $K$. The fully discretized problem read then as: Find $u_{h}^{n} \in V_{h}^{p}$ such that

$$
C_{m} \int_{\mathcal{T}_{h}} \frac{u_{h}^{n}-u_{h}^{n-1}}{d t} \varphi_{h} \mathrm{~d} \mathbf{x}+\frac{1}{\chi} \int_{\mathcal{T}_{h}} \mathbf{D} \nabla u_{h}^{n} \cdot \nabla \varphi_{h} \mathrm{~d} \mathbf{x}=\sum_{K} \tilde{I}_{K}^{n-1}\left(u_{h}^{n-1}, \varphi_{h}\right)
$$

for all $\varphi_{h} \in V_{h}^{p}$, and with $I_{K}$ given, resulting from the approximation of the integral

$$
\tilde{I}_{K}^{n-1}(v, \phi) \approx I_{K}^{n-1}(v, \phi):=\int_{K} I_{i o n}\left(v, \mathbf{w}^{n-1}\right) \phi \mathrm{d} \mathbf{x}, \quad v, \phi: \Omega \rightarrow \mathbb{R} .
$$

In literature there exist different methods to compute this approximation, which result in different spatial discretizations of the gating variables $\mathrm{w}^{n}$. Since these can indeed be applied to any type of Galerkin scheme, our specific choice is detailed in Section 2.5

\subsection{Hybridizable Discontinuous Galerkin approximation}

The derivation of the HDG approximation is based on the work of Nguyen et al. [30] for the linear convection-diffusion equation. We will briefly introduce its derivation, starting from the strong form 
of the mixed formulation for the diffusion term of Equation (2), i.e.,

$$
\left\{\begin{aligned}
C_{m} \frac{u^{n}-u^{n-1}}{d t}-\frac{1}{\chi} \nabla \cdot \mathbf{q}^{n} & =I_{\text {ion }}\left(u^{n-1}, \mathbf{w}^{n-1}\right) \\
\mathbf{q}^{n}-\mathbf{D} \nabla u^{n} & =0
\end{aligned}\right.
$$

and its weak form on the element $K$

$$
\left\{\begin{aligned}
C_{m} \int_{K} \frac{u^{n}-u^{n-1}}{d t} \varphi \mathrm{d} \mathbf{x}+\frac{1}{\chi} \int_{K} \mathbf{q}^{n} \cdot \nabla \varphi \mathrm{d} \mathbf{x}-\frac{1}{\chi} \int_{\partial K} \mathbf{n} \cdot \hat{\mathbf{q}}^{n} \varphi \mathrm{d} \mathbf{x}=I_{K}^{n-1}\left(u^{n-1}, \varphi\right), \\
\int_{K} \mathbf{D}^{-1} \mathbf{q}^{n} \cdot \boldsymbol{\psi} \mathrm{d} \mathbf{x}+\int_{K} u \nabla \cdot \boldsymbol{\psi} \mathrm{d} \mathbf{x}-\int_{\partial K} \lambda^{n} \mathbf{n} \cdot \boldsymbol{\psi} \mathrm{d} \mathbf{x}=0,
\end{aligned}\right.
$$

for all $\varphi \in L_{2}(K), \psi \in\left[L_{2}(K)\right]^{d}$. Here, $\lambda^{n} \in L_{2}(e)$ is the single-valued trace of $u^{n}$ over the face $e \in \mathcal{E}_{h}$. $\mathcal{E}_{h}$ is the set of all interior and boundary faces, where an interior face is defined as $e=\partial K_{1} \cap \partial K_{2}$ between two elements $K_{1}$ and $K_{2}$ and a boundary face is defined as $e=\partial K \cap \partial \Omega$ for an element $K$. The vector $\hat{\mathbf{q}}^{n}$ is the trace on $\partial K$ of $\mathbf{q}^{n}$, which is defined in the HDG method by:

$$
\hat{\mathbf{q}}^{n}=\mathbf{q}^{n}-\tau\left(u^{n}-\lambda^{n}\right) \mathbf{n} \text { on } \partial K,
$$

with the stabilization parameter $\tau>0$.

Finally, we close the system by enforcing the jump across of the trace of the flux $\hat{\mathbf{q}}$ along each edge $e$ to be zero, i.e.,

$$
\left[\left[\hat{\mathbf{q}}^{n}\right]\right]=\mathbf{0} \text {, on all } e \in \mathcal{E}_{h} .
$$

For the spatial discretization, we define the finite element spaces of piecewise discontinuous polynomials on the element's volume

$$
W_{h}^{p}=\left\{v \in L^{2}(\Omega):\left.v\right|_{K} \in \mathcal{P}^{p}(K) \text { for element } K \in \mathcal{T}_{h}\right\}
$$

and on the elements edges

$$
M_{h}^{p}=\left\{\mu \in L^{2}\left(\mathcal{E}_{h}\right):\left.\mu\right|_{e} \in \mathcal{P}^{p}(e) \text { for face } e \in \mathcal{E}_{h}\right\} .
$$

With these new definitions we can now formulate the fully discretized system of equations corresponding to the HDG method: Find $\left(u_{h}^{n}, \mathbf{q}_{h}^{n}, \lambda_{h}^{n}\right) \in W_{h}^{p} \times\left[W_{h}^{p}\right]^{d} \times M_{h}^{p}$ such that:

$$
\left\{\begin{array}{r}
\sum_{K}\left(\int_{K} C_{m} \frac{u_{h}^{n}-u_{h}^{n-1}}{d t} \varphi_{h} \mathrm{~d} \mathbf{x}+\frac{1}{\chi} \int_{K} \mathbf{q}_{h}^{n} \nabla \varphi_{h} \mathrm{~d} \mathbf{x}-\frac{1}{\chi} \int_{\partial K} \mathbf{q}_{h}^{n} \cdot \mathbf{n} \varphi_{h}-\tau\left(u_{h}^{n}-\lambda_{h}^{n}\right) \varphi_{h} \mathrm{~d} \mathbf{x}\right) \\
=\sum_{K} \tilde{I}_{K}^{n-1}\left(u_{h}^{n-1}, \varphi_{h}\right) \\
\sum_{K}\left(\int_{K} \mathbf{D}^{-1} \mathbf{q}_{h}^{n} \cdot \boldsymbol{\psi}_{h} \mathrm{~d} \mathbf{x}+\int_{K} u_{h}^{n} \nabla \cdot \boldsymbol{\psi}_{h} \mathrm{~d} \mathbf{x}-\int_{\partial K} \lambda_{h}^{n} \mathbf{n} \cdot \boldsymbol{\psi}_{h} \mathrm{~d} \mathbf{x}\right) \\
\sum_{K}\left(\int_{\partial K} \mathbf{q}_{h}^{n} \cdot \mathbf{n} \mu_{h} \mathrm{~d} \mathbf{x}-\int_{\partial K} \tau\left(u_{h}^{n}-\lambda_{h}^{n}\right) \mu_{h} \mathrm{~d} \mathbf{x}\right)
\end{array}\right.
$$

for all $\left(v_{h}, \boldsymbol{\psi}_{h}, \mu_{h}\right) \in W_{h}^{p} \times\left[W_{h}^{p}\right]^{d} \times M_{h}^{p}$.

One of the advantages of HDG methods is that the size of the final linear system can be considerably reduced using its structure. First, let us rewrite the system of equations as follows

$$
\left\{\begin{aligned}
m\left(u_{h}^{n}, \varphi_{h}\right) / d t-b\left(\varphi_{h}, \mathbf{q}_{h}^{n}\right)+a\left(u_{h}^{n}, \varphi_{h}\right)-c\left(\lambda_{h}^{n}, \varphi_{h}\right) & =f^{n-1}\left(\varphi_{h}\right) \\
d\left(\mathbf{q}_{h}^{n}, \boldsymbol{\psi}_{h}\right)+b\left(u_{h}^{n}, \boldsymbol{\psi}_{h}\right)-e\left(\lambda_{h}^{n}, \boldsymbol{\psi}_{h}\right) & =0 \\
e\left(\mu_{h}, \mathbf{q}_{h}^{n}\right)-c\left(\mu_{h}, u_{h}^{n}\right)+h\left(\lambda_{h}^{n}, \mu_{h}\right) & =0
\end{aligned}\right.
$$


with the integral forms given by

$$
\begin{aligned}
m(u, \varphi) & =\sum_{K} \int_{K} C_{m} \chi u \varphi \mathrm{d} \mathbf{x}, & d(\mathbf{q}, \boldsymbol{\psi}) & =\sum_{K} \int_{K} \mathbf{D}^{-1} \mathbf{q} \cdot \boldsymbol{\psi} \mathrm{d} \mathbf{x}, \\
a(u, \varphi) & =\sum_{K} \int_{\partial K} \tau u \varphi \mathrm{d} \mathbf{x}, & e(\lambda, \boldsymbol{\psi}) & =\sum_{K} \int_{\partial K} \lambda \boldsymbol{\psi} \cdot \mathbf{n} \mathrm{d} \mathbf{x}, \\
b(u, \boldsymbol{\psi}) & =\sum_{K} \int_{K} u \nabla \cdot \boldsymbol{\psi} \mathrm{d} \mathbf{x}, & f^{n-1}(\varphi) & =m\left(u_{h}^{n-1}, \varphi\right) / d t+\chi \sum_{K} \tilde{I}_{K}^{n-1}\left(u_{h}^{n-1}, \varphi\right), \\
c(\lambda, \varphi) & =\sum_{K} \int_{\partial K} \varphi \tau \lambda \mathrm{d} \mathbf{x}, & h(\lambda, \mu) & =\sum_{K} \int_{\partial K} \tau \lambda \mu \mathrm{d} \mathbf{x},
\end{aligned}
$$

for all $(\mathbf{q}, u, \lambda)$ and $(\varphi, \psi, \mu)$ in $\left(V_{h}^{p} \times W_{h}^{p} \times M_{h}^{p}\right)$.

Denoting the vectors of degrees of freedom $U^{n}, Q^{n}, \Lambda^{n}$ associated to $u_{h}^{n}, \mathbf{q}_{h}^{n}, \lambda_{h}^{n}$, respectively, we can write the resulting system in matrix form as:

$$
\left[\begin{array}{c}
M / d t U^{n} \\
0 \\
0
\end{array}\right]+\left[\begin{array}{ccc}
A & B & -C \\
-B^{T} & D & -E \\
-C^{T} & E^{T} & H
\end{array}\right]\left[\begin{array}{c}
U^{n} \\
Q^{n} \\
\Lambda^{n}
\end{array}\right]=\left[\begin{array}{c}
F^{n-1} \\
0 \\
0
\end{array}\right]
$$

Since the matrices $A, B, D$ do no couple between different elements, we can use static condensation (Schur complements) on the element level to reduce the global system size [27]. For that purpose, we eliminate the interior variables $U^{n}$ and $Q^{n}$ in an element-by-element fashion, so that the remaining degrees of freedom are those of the trace variable $\Lambda^{n}$ on the faces of $\mathcal{E}_{h}$.

\subsection{Ionic current approximation}

As stated above, there exist several methods to approximate the element integral of the nonlinear ionic current term $I_{i o n}$ in Galerkin formulations for cardiac electrophysiology

$$
\tilde{I}_{K}\left(u_{h}, \varphi_{h}\right) \approx \int_{K} I_{i o n}\left(u_{h}, \mathbf{w}\right) \varphi_{h} \mathrm{~d} \mathbf{x}
$$

with both $u_{h}$ and $\varphi_{h}$ given through spatial polynomial functions of degree $p$, see e.g. [7, 9, 10] and references therein.

In this work, we adopt a numerical approximation of $I_{K}$ by defining one gating variable at each quadrature point $\mathbf{x}_{\ell}$ within the element $K$, namely

$$
\tilde{I}_{K}\left(u_{h}, \varphi_{h}\right)=\sum_{\ell=1}^{b_{K}} \alpha_{\ell} I_{i o n}\left(u_{h}\left(\mathbf{x}_{\ell}\right), \mathbf{w}_{\ell}\right) \varphi_{h}\left(\mathbf{x}_{\ell}\right)
$$

with $\alpha_{\ell}$ the quadrature weights and $b_{K}$ the number of integration points on the element $K$.

This strategy allows to represent the ionic term at a higher degree compared with the ionic current interpolation (see e.g. [10]), where the ionic current is discretized using the same space as for the potential. Note that in both the HDG and CG cases the number of quadrature points to build the term $\tilde{I}_{K}$ are the same, for a given polynomial order $p$.

In this work, the number of quadrature points $b_{K}$ is chosen depending on the polynomial degree $p$ of the finite element spaces on element $K$. Concretely, assuming that $I_{i o n}$ has the potential $u$ involving polynomial expressions in $u$ of degree up to $k_{i o n}$, we propose to choose the quadrature order to integrate exactly polynomials of degree $p\left(k_{i o n}+1\right)$, since the integral in the weak form comprises not only $I_{i o n}(u)$ but also the test function. For the integration rule we do not take into account the additional non-polynomial terms in the model, e.g. the Heaviside function. Note that for high-order curved geometry descriptions there could still be a small integration error, but it is expected to be of higher order than the discretization error. For the numerical examples we use hexahedral and tetrahedral elements with appropriate intergration rules. An example will be shown in Section 3.2. Note that keeping track of $\mathbf{w}_{\ell}$ and $I_{i o n}$ in quadrature points can be interpreted as a polynomial approximation of at least degree $\left\lceil\left(\left(k_{i o n}+1\right) p+1\right) / 2\right\rceil-1$ in the discontinuous setting by Lagrange polynomials through the integration points. 
We want to remark that other ionic current approximations could also be used, e.g. where the gating variables are stored at the degrees of freedom of the finite element approximation of the potential. However, additionally investigating the effect of the type of ionic current approximation is out of the scope of this article.

\section{6. p-adaptivity for $H D G$}

The HDG method allows for a straightforward adaptation of the degree of the polynomial basis functions since different polynomial degrees between neighboring elements can be independently chosen. A key ingredient is to apply a proper error indicator for selecting the local polynomial order. In this work we choose a simplified version of the error indicator presented in [23] based on the jump of the gradient of the potential across the face $\gamma$. Therefore, on each element $K$ the error indicator is defined via the numerical gradient on the faces $\hat{\mathbf{q}}$ as

$$
e_{K}^{n}=\sum_{\gamma \in \partial K}\left\|\hat{\mathbf{q}}_{\gamma}^{n} \cdot \mathbf{n}\right\|_{L_{2}(\gamma)}^{2} \frac{1}{A_{\gamma}}
$$

with $A_{\gamma}$ the surface area of the face. Then the new degree on the element is calculated via

$$
p_{K}^{n}=p_{K}^{n-1}+\left\lceil\frac{1}{\omega} \ln \left(\frac{e_{K}^{n-1}}{e_{t o l}}\right)\right\rceil
$$

for a constant $\omega=1.66$ according to [23], which is estimated from convergence rates of high-order $\mathrm{CG}$ elements, and a given error tolerance $e_{t o l}$. This value did lead to satisfactory results, that also were quite insensitive to slight variations.

The adaption of the polynomial degree is calculated for each element independently, while the polynomial degree of the face is defined by the higher of the two polynomial degrees of the elements sharing this face.

\section{NUMERICAL EXPERIMENTS}

\subsection{Academic problem setup}

In our test we use a modified version of the problem setup from [3]. The geometry corresponds to a myocardial tissue cuboid of size $12 \mathrm{~mm} \times 4 \mathrm{~mm} \times 2 \mathrm{~mm}$, see Figure 1 a. To start the propagation of the electrochemical wave we use the initial condition

$$
u(\mathbf{x}, 0)=0.5(1-\tanh (1000(x-2))),
$$

shown in Figure $1 \mathrm{~b}$.

Given that our study focuses on the investigation of different spatial approximations of the problem, we restrict the cardiac electrophysiology problem to the model of Bueno-Orovio et al. [31], which is able to reproduce important physiological properties, e.g. action potential curves and upstroke velocities, with only three gating variables and three ionic currents. The model is given as

$$
\begin{aligned}
\partial_{t} u & =\nabla \cdot(\sigma \nabla v)-I_{i o n}(u, \mathbf{w}), \\
\partial_{t} w_{1} & =\left(1-H\left(u-\theta_{w_{1}}\right)\right)\left(w_{1, \infty}-w_{1}\right) / \tau_{w_{1}}^{-}-H\left(u-\theta_{w_{1}}\right) w_{1} / \tau_{w_{1}}^{+}, \\
\partial_{t} w_{2} & =\left(1-H\left(u-\theta_{w_{2}}\right)\right)\left(w_{2, \infty}-w_{2}\right) / \tau_{w_{2}}^{-}-H\left(u-\theta_{w_{2}}\right) w_{2} / \tau_{w_{2}}^{+}, \\
\partial_{t} w_{3} & \left.=\left(1+\tanh \left(k_{w_{3}}\left(u-u_{w_{3}}\right)\right)\right) / 2-w_{3}\right) / \tau_{w_{3}},
\end{aligned}
$$

with $H(\cdot)$ the Heaviside function and with the computation of the ionic current $I_{i o n}$ as

$$
\begin{aligned}
I_{i o n}(u, \mathbf{w}) & =I_{f i}+I_{s o}+I_{s i}, \\
I_{f i} & =-w_{1} H\left(u-\theta_{w_{1}}\right)\left(u-\theta_{w_{1}}\right)\left(u_{u}-u\right) / \tau_{f i}, \\
I_{s o} & =\left(u-u_{0}\right)\left(1-H\left(u-\theta_{w_{2}}\right)\right) / \tau_{0}+H\left(u-\theta_{w_{2}}\right) \tau_{s o}, \\
I_{s i} & =-H\left(u-\theta_{w_{2}}\right) w_{2} w_{3} / \tau_{s i} .
\end{aligned}
$$




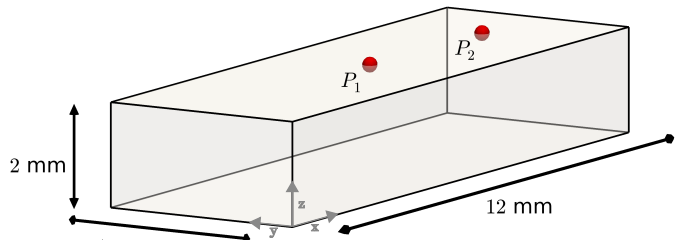

(a)

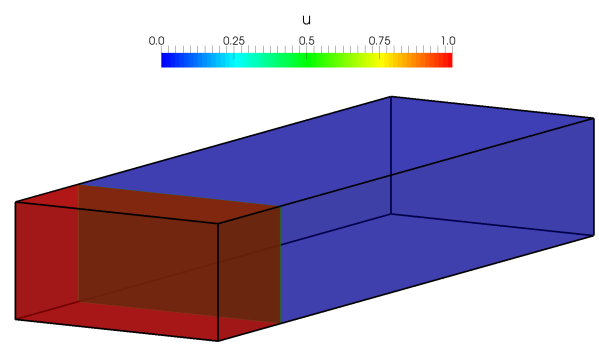

(b)

Figure 1. Myocardial tissue slab. (a) Rectangular domain $(12 \mathrm{~mm} \times 4 \mathrm{~mm} \times 2 \mathrm{~mm})$. The time difference in activation is measured between the two red points $P_{1}$ and $P_{2}\left(x_{1}=6 \mathrm{~mm}, y_{1}=2 \mathrm{~mm}, z_{1}=2 \mathrm{~mm}\right.$ and $x_{2}=10 \mathrm{~mm}, y_{2}=2 \mathrm{~mm}, z_{2}=2 \mathrm{~mm}$ ). (b) Initial condition.

\begin{tabular}{ccccccc}
\hline$u_{o}$ & $u_{u}$ & $\theta_{v}$ & $\theta_{w}$ & $\theta_{v}^{-}$ & $\theta_{o}$ & $\tau_{v 1}^{-}$ \\
0 & 1.55 & 0.3 & 0.13 & 0.006 & 0.006 & 60 \\
\hline \hline$\tau_{v 2}^{-}$ & $\tau_{v}^{+}$ & $\tau_{w 1}^{-}$ & $\tau_{w 1}^{-}$ & $K_{w}^{-}$ & $u_{w}-$ & $\tau_{w}^{+}$ \\
1150 & 1.4506 & 60 & 15 & 65 & 0.03 & 200 \\
\hline \hline$\tau_{f i}$ & $\tau_{o 1}$ & $\tau_{o 2}$ & $\tau_{s o 1}$ & $\tau_{s o 2}$ & $k_{s o}$ & $u_{s o}$ \\
0.11 & 400 & 6 & 30.0181 & 0.9957 & 2.0458 & 0.65 \\
\hline \hline$\tau_{s 1}$ & $\tau_{s 2}$ & $k_{s}$ & $u_{s}$ & $\tau_{s i}$ & $\tau_{w_{\infty}}$ & $w_{\infty}^{*}$ \\
2.7342 & 16 & 2.0994 & 0.9087 & 1.8875 & 0.07 & 0.94 \\
\hline
\end{tabular}

Table I. Epicardial model parameter values.

and with the definitions of the functions

$$
\begin{aligned}
\tau_{v}^{-} & =\left(1-H\left(u-\theta_{v}^{-}\right)\right) \tau_{v_{1}}^{-}+H\left(u-\theta_{v}^{-}\right) \tau_{v_{2}}^{-}, \\
\tau_{w}^{-} & =\tau_{w_{1}}^{-}+\left(\tau_{w_{2}}^{-}-\tau_{w_{1}}^{-}\right)\left(1-\tanh \left(k_{w}^{-}\left(u-u_{w}^{-}\right)\right)\right) / 2, \\
\tau_{s o} & =\tau_{s o 1}+\left(\tau_{s o 2}-\tau_{s o 1}\right)\left(1-\tanh \left(k_{s o}\left(u-u_{s o}\right)\right)\right) / 2, \\
\tau_{s} & =\left(1-H\left(u-\theta_{w}\right)\right) \tau_{s_{1}}+H\left(u-\theta_{w}\right) \tau_{s 2}, \\
\tau_{s} & =\left(1-H\left(u-\theta_{o}\right)\right) \tau_{o_{1}}+H\left(u-\theta_{o}\right) \tau_{o 2}, \\
w_{\infty} & =\left(1-H\left(u-\theta_{o}\right)\right)\left(1-u / \tau_{w_{\infty}}+H\left(u-\theta_{o}\right) w_{\infty}^{*},\right. \\
v_{\infty} & = \begin{cases}1 & u<\theta_{v}^{-} \\
0 & u \geqslant \theta_{v}^{-}\end{cases}
\end{aligned}
$$

The parameter values, which can be adapted to model different cell types, can be found in the original work [31]. In the present study we choose the epicardial parameter values (see Table I]. If not indicated otherwise, the diffusion coefficient is chosen as $\sigma=0.1 \mathrm{~mm}^{2} / \mathrm{ms}$. The time step is chosen as $\Delta t=0.1 \mathrm{~ms}$, and different mesh sizes are used as indicated for each experiment.

To compare the results we calculate the conduction velocity $c v$ in all simulations by measuring the activation times $(\operatorname{acttime}(\cdot))$ of point $P_{1}(6,2,2)$ and point $P_{2}(10,2,2)$ (see Figure 1 1 $)$ ) and with the distance between the two points to obtain the conduction velocity via $c v=\| P_{2}-$ $P_{1} \| /\left(\operatorname{acttime}\left(P_{2}\right)-\operatorname{acttime}\left(P_{1}\right)\right)$. We define the activation time as the time when the voltage is larger than $1.7 \mathrm{mV}$, which is equivalent to the value of 1 for the dimensionless variable $u$ in the chosen cellular model. The converged conduction velocity amounts to around $0.73 \mathrm{~mm} \mathrm{~ms}^{-1}$.

\subsection{Choice of the ionic current integration formula}

Here we briefly describe the application of the rule defined in Section 2.5] to the ionic model [31]. We identify the maximal polynomial degree of $u$ in the model as $k_{i o n}=2$ through $I_{f i}$. Therefore, 
we choose the number of quadrature points $b_{K}$ such that polynomials of degree $d$

$$
d=\left(k_{i o n}+1\right) p=3 p
$$

are exactly integrated.

We need a definition of an integration rule for the ionic current term that varies with the polynomial order. This necessity is visualized in Figure 2 for $\sigma=0.1 \mathrm{~mm} \mathrm{~ms}^{-2}$ and $\tau=1 \mathrm{~mm} \mathrm{~ms}^{-1}$ and $h=1 \mathrm{~mm}$. We observe, in general, two types of issues arising when a fixed number of integration points is chosen. The first issue is a propagation block, which means that no propagation takes place. This could happen if the ionic flow from the neighboring element is not sufficient to reach the threshold for the self depolarization in an element. It occurs in our examples when the polynomial degree is increased above a certain value, see e.g. the case $b_{K}=1,8,27$ in Figure 2 . For one integration point $\left(b_{K}=1\right)$ only at polynomial degrees zero and one the propagation takes place. For $b_{K}=8$ propagation takes place until degree three and for $b_{K}=27$ no propagation occurs for degree six, eight, nine, twelve and thirteen. The second issue is observed with $b_{K}=27$ where the interaction between different computational errors, for example the insufficient integration of the ionic current term and coarse meshes, could leads to a propagation for some polynomial degrees $(6,10$ and 11) but with wrong results. This is the second issue. In the above mentioned cases for $b_{K}=27$ and every time for $b_{K}=64$ for high polynomial degrees a propagation takes place but the computed conduction velocity is wrong, since the error in the computation of the ionic current dominates. This can be seen in the oscillating behavior of the computed conduction velocity for increasing polynomial degree (see Figure 2 a).

Note that considerably increasing the number of integration points (e.g. $b_{K}=8000$ ) leads to a smooth convergence curve with respect to an increase of the polynomial order. However, using too many integration points results in an increased computational cost without a significant gain in the accuracy of the results. Finally, the adaptation of $b_{K}$ in dependence of the polynomial order as defined in Equation (19) is able to give convergence at a much lower cost (see Figure 2p). However, for polynomial degree one for the adapted integration points, the conduction velocity is lower than expected. This is due to the additional non-polynomial terms in the model in the calculation of the ionic current term. For low order $p=1$, we experimentally determined that using integration exact to degree 3 is not accurate enough. Thus, we tested a stricter rule for the integration points so that polynomials of degree $d$

$$
d=\left(k_{i o n}+2\right) p=4 p
$$

are exactly integrated. This stricter rule (Equation 20 ) solves the problem with low polynomial degrees but it has almost no influence for the computation with higher polynomial degrees (see Figure $2 \mathrm{p}$ ). Hence, to reduce the computational cost for high polynomial degrees we decided to use the rule from Equation (19) for high order polynomials, but for $p=1$ we use the rule defined in Equation (20) so that in any case at least polynomials of degree 4 are exactly integrated, i.e.

$$
d=\left\{\begin{array}{ll}
3 p & , p>1 \\
4 p & , p \leq 1
\end{array} .\right.
$$

\subsection{Choice of the stabilization parameter}

In this section we study the influence of the HDG stabilization parameter $\tau$ on the solution quality. First, from Equation (7) we can observe that a large stabilization parameter $\tau \rightarrow \infty$ forces the solution $u_{h}$ to be continuous, therefore reproducing the CG solution. This is verified in Figure 3 , where the solutions are almost identical and thus the curves are the same.

Figure 4 presents results for different electrical conductivities $\sigma$ in a wide range of values of physiological interest [2, 32]. Firstly, we can see that with increasing polynomial order $p$ the influence of the stabilization parameter decreases. In Figure 4 only $h=0.5 \mathrm{~mm}$ is plotted since propagation for $h=2 \mathrm{~mm}$ takes place only for larger polynomial degrees. In all plots in Figure 


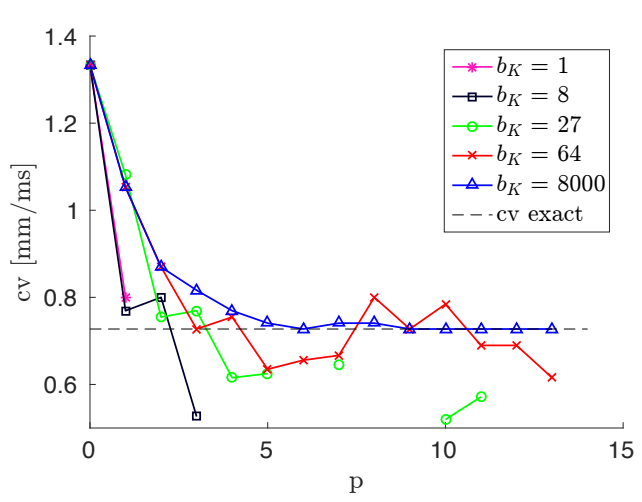

(a)

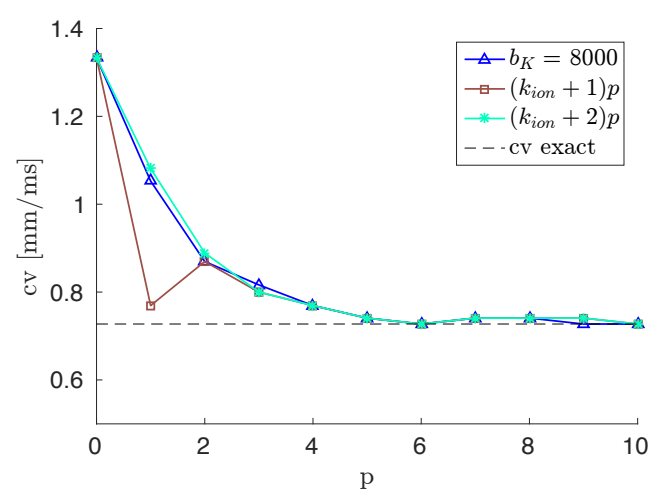

(b)

Figure 2. Conduction velocity plotted over the polynomial degree $p$ for varying number of integration points $b_{K}$. (a) Comparison between different constant numbers of integration points. (b) Comparison between adapted number of integration points and a large constant number.

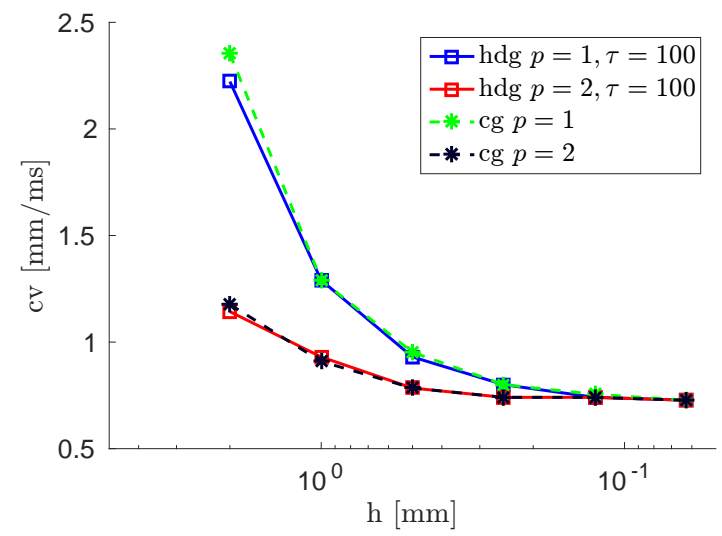

Figure 3. Conduction velocity (cv) plotted over the element size $h$ for $\sigma=0.1 \mathrm{~mm}^{2} / \mathrm{ms}$. The HDG solution for large $\tau$ is the same as the CG solution.

4 it is visible that for larger $\tau$, the conduction velocity is overestimated and for smaller $\tau$ it is underestimated. A small $\tau$ could also lead to the case that no propagation takes place. This propagation block can be seen in the plots, when no value for the conduction velocity is plotted. Decreasing the element size also leads to a decrease of the influence of the stabilization parameter. Nevertheless, over- and underestimation is still visible for high and low stabilization parameters, respectively.

In Figure 5 the results for a more detailed range of $\tau=0.1$ to $1 \mathrm{~mm} / \mathrm{ms}(5 \mathrm{k})$ and $\tau=1$ to $10 \mathrm{~mm} / \mathrm{ms}(5 \mathrm{~b})$ are plotted. We conclude that a reasonable value for the stabilization parameter $\tau$ for a wide range of physiological diffusivities and element sizes is $\tau=1 \mathrm{~mm} / \mathrm{ms}$ using the minimal cell model [31]. For other cell models the behavior of the stabilization parameter can be easily tested in a similar fashion. Note that one should not use too low polynomial degrees to ensure only a small dependence on $\tau$.

\subsection{Time discretization}

For the sake of completeness, we briefly present the results of a time step refinement for a polynomial order of $p=2$. In Figure 6 one can see the conduction velocity for different combinations of spatial and temporal refinement. For temporal refinement we consider time steps of 


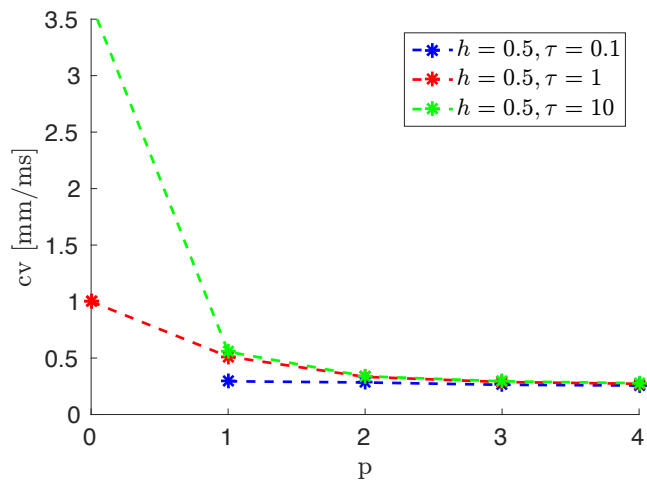

(a) $\sigma=0.01 \mathrm{~mm}^{2} / \mathrm{ms}$

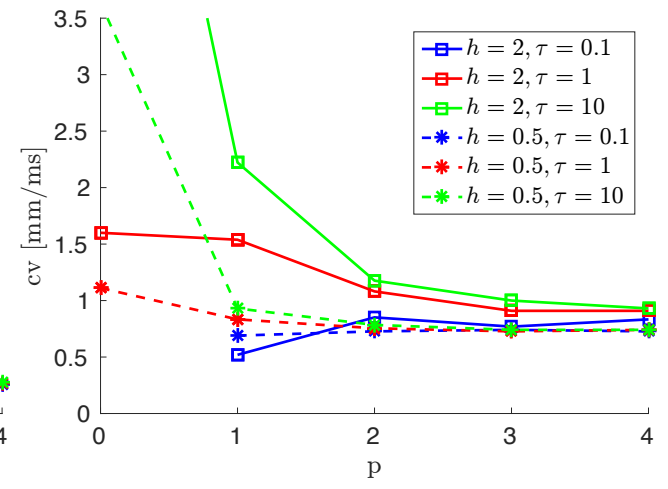

(b) $\sigma=0.1 \mathrm{~mm}^{2} / \mathrm{ms}$

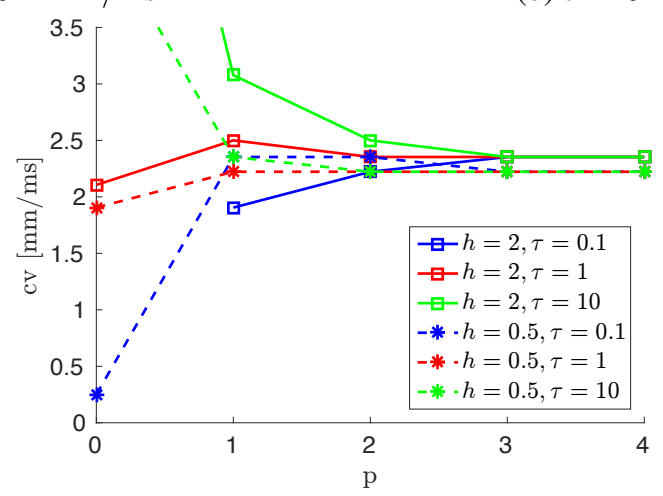

(c) $\sigma=1 \mathrm{~mm}^{2} / \mathrm{ms}$

Figure 4. Conduction velocities for varying HDG stabilization parameters $\tau$ and diffusion coefficients $\sigma$.

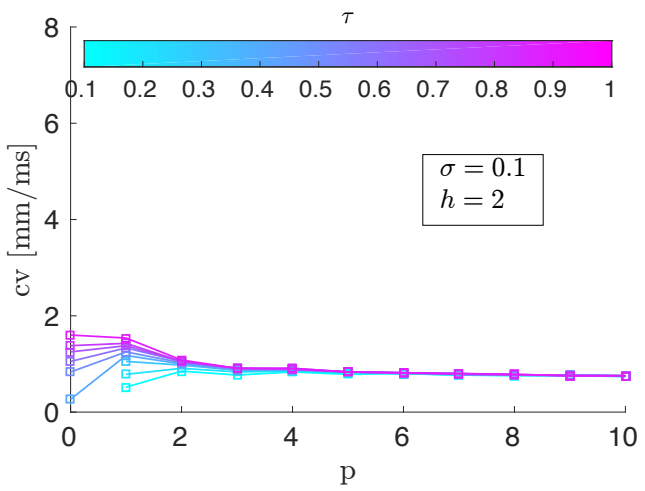

(a) $\tau=0.1$ to $1 \mathrm{~mm} / \mathrm{ms}$

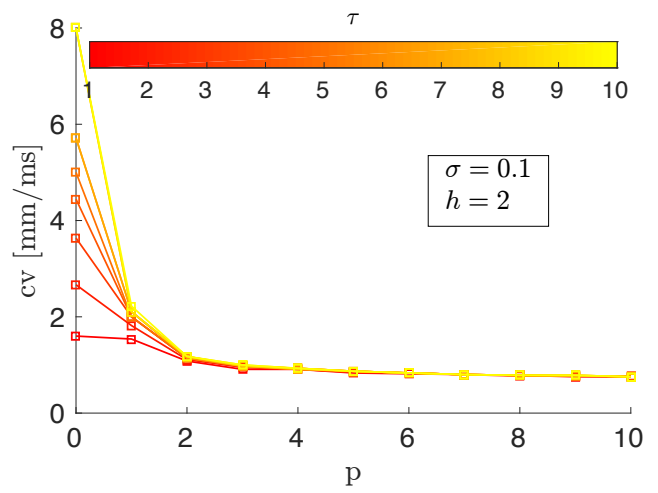

(b) $\tau=1$ to $10 \mathrm{~mm} / \mathrm{ms}$

Figure 5. Detailed analysis of the influence of the stabilization parameter $\tau$ for $\sigma=0.1$.

$\Delta t=10^{-1}, 10^{-2}, 10^{-3}$ and $10^{-4} \mathrm{~ms}$ and for spatial refinement element sizes of $h=2,1,0.5,0.25$ and $0.125 \mathrm{~mm}$. With a time step of $\Delta t=0.1 \mathrm{~ms}$ the solution is not yet fully converged in terms of temporal errors. However, decreasing the time step does not change the convergence behavior of the general solution. Thus, analyzing different spatial discretization methods can be done on a coarser time step, without missing important characteristics of the solution. Figure 6 shows that with the 


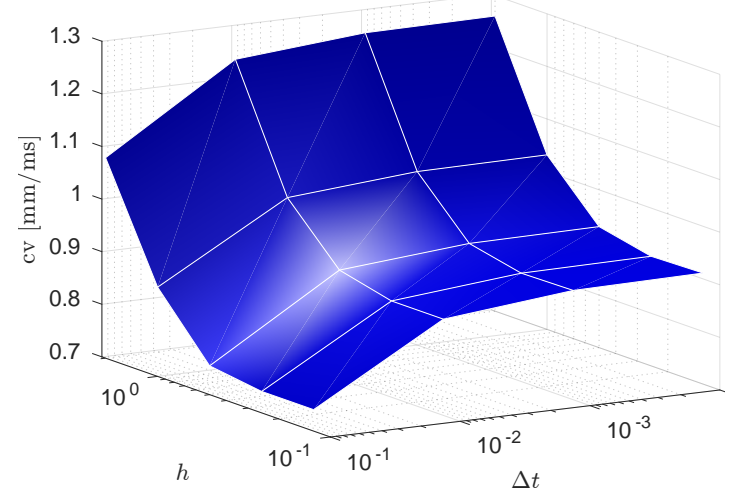

Figure 6. Conduction velocity plotted over the element size $h$ and the time step $\Delta t$ for a polynomial order of $p=2$. The range from the element size is $h=2,1,0.5,0.25$ and $0.125 \mathrm{~mm}$ and the time steps are $\Delta t=$ $10^{-1}, 10^{-2}, 10^{-3}$ and $10^{-4} \mathrm{~ms}$.

semi-implicit time discretization, the conduction velocity is overestimated for a coarse mesh size, while for a large time step it is underestimated.

\subsection{Convergence and efficiency analysis with $h$ and $p$ refinement}

Next we analyze the change in conduction velocity when decreasing the element size and increasing polynomial order for the HDG method for both tetrahedral and hexahedral meshes. Additionally, we compare the HDG discretization method with CG discretizations in more detail. For the HDG method we use a stabilization parameter $\tau=1 \mathrm{~mm} \mathrm{~ms}^{-1}$ (see Section 3.3. For both methods, HDG and $\mathrm{CG}$, the rule for the integration points for the ionic current is adapted to the polynomial degree as defined in Sections 2.5 and 3.2

To compare the results we record the number of degrees of freedom (ndof) and the number of non-zeros in the system matrix (nnz), since they can be seen as the key factors for the calculation time [26]. For the CG method, we consider these quantities directly from the discrete system for the potential $u_{h}$. In HDG we do it for the condensed system involving only the trace variables $\Lambda_{h}$. We want to point out that for low order, the linear systems for HDG have more unknowns than for CG while for high order, HDG has less unknowns than CG, i.e., the unknowns for HDG increases more slowly with increasing order [26].

First, we compare the HDG and CG methods for hexahedral meshes. Both methods appear to deliver similar results in terms of precision versus ndof and nnz (see Figure 7). It is also evident that both ways, i.e., to increase the degree or to reduce the mesh size, are valid to better approximate the conduction velocity.

Additionally, our results suggest that to achieve a converged conduction velocity for HDG and $\mathrm{CG}$ and independent of the mesh type, approximately the same number of degrees of freedom and non-zero entries in the system matrix are needed, except for a polynomial order $p=0$ in HDG. For the ndof this can be seen in Figure $7 \mathrm{a}$ where the polynomial order is kept fixed and the element size is decreasing, and more clearly in Figure 7k, where the element size is kept fixed and polynomial order is increasing, and similarly for the nnz in Figure $7 \mathrm{p}$ and Figure $7 \mathrm{~d}$.

For the same number of degrees of freedom, if the result is approximated with high-order elements instead of small sized lower order elements, the obtained conduction velocity is closer to the exact solution (see Figure $8 \mathrm{a}$ ). In terms of number of nnz, increasing the polynomial order or decreasing the element size leads to the same result (see Figure $8 \mathrm{p}$ ). Thus, the HDG method becomes increasingly competitive as the polynomial degree is increased. For $h=0.5$ the conduction velocity increases slightly at one point, although the conduction velocity should decrease due to the general convergence behavior. The slight increase could happen since we measure only a scalar value to look for the convergence of the solution. A coarse time step, a coarse mesh and the averaging of the 

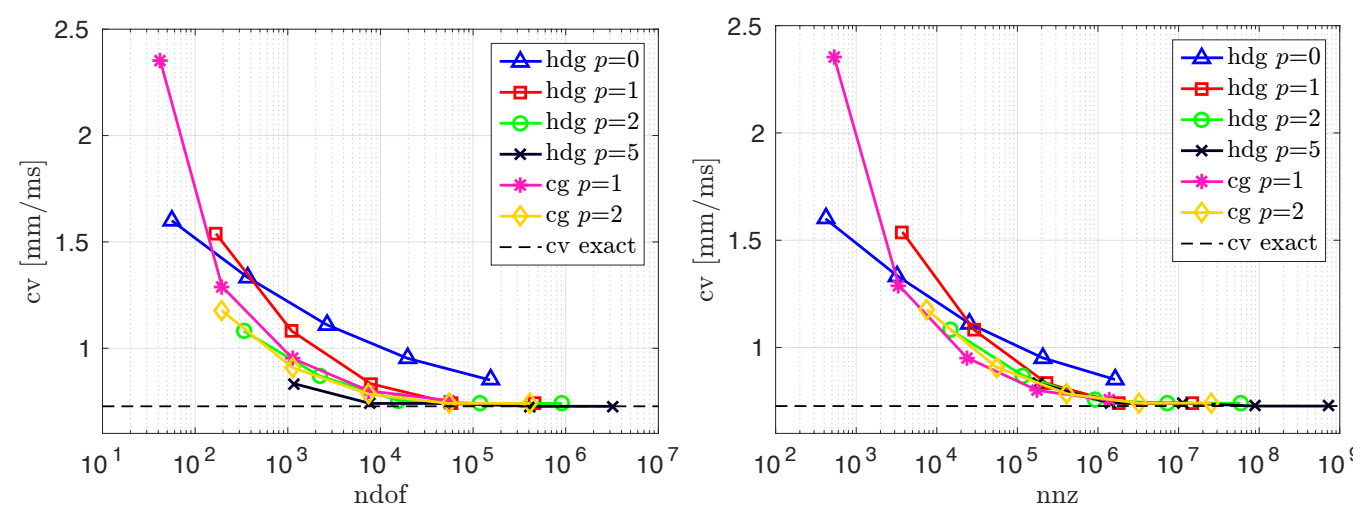

(a) number of degrees of freedom (ndof) via $h$ refinement

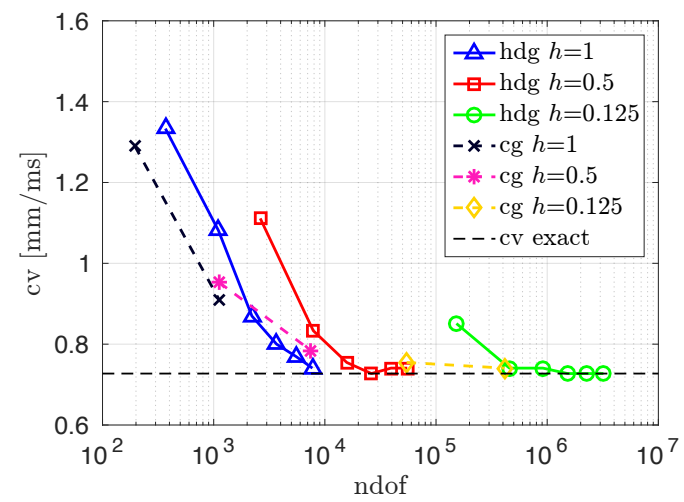

(b) number of non-zeros in system matrix (nnz) via $h$-refinement

(c) number of degrees of freedom (ndof) via $p$ refinement

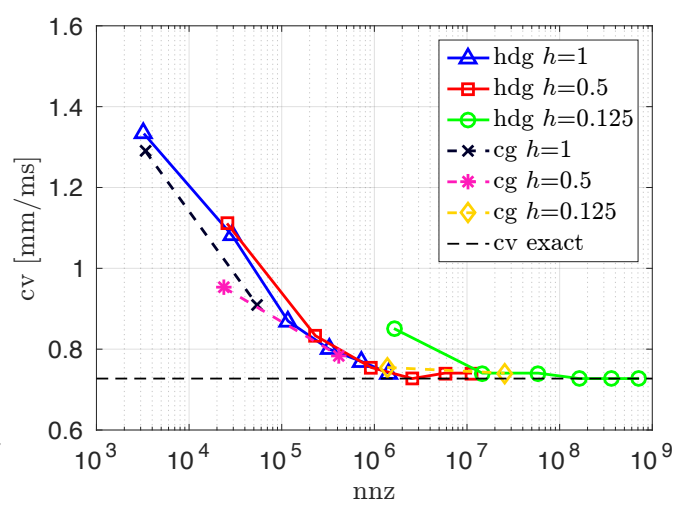

(d) number of non-zeros in system matrix (nnz) via $p$-refinement

Figure 7. Conduction velocity plotted over ndof and nnz. (a) and (b) show the conduction velocity for the element sizes $h=2,1,0.5,0.25$ and $0.125 \mathrm{~mm}$. (c) and (d) show the conduction velocity for polynomial order $p=0,1,2,3,4$ and 5 for HDG and $p=1$ and 2 for CG.

values around the measuring point could lead to small rounding errors, which cause a slight change in the computed conduction velocity. However, the convergence behavior of the overall problem does not change.

In Figure 9, we compare tetrahedral with hexahedral meshes. Note that for a fixed $h$, tetrahedral meshes involve a larger number of elements than hexahedral meshes. Although for a fixed $h$ in CG both mesh discretizations, hexahedral and tetrahedral, deliver the same ndof in the structured mesh, in HDG (and particularly high order) the tetrahedral mesh involves a considerably larger number of degrees of freedom. However, we can clearly appreciate that we roughly require the same number of degrees of freedom and nnz with tetrahedral and hexahedral meshes for achieving similar precision, i.e., the precision is only depending on the ndof and is independent of the element type. Note that for high-order tetrahedral elements we use optimized nodal sets, the so-called warp and blend points, to avoid ill-conditioned interpolation and negative impact on linear solvers [33].

\subsection{Results for p-adaptivity}

The previous results show that increasing the polynomial order in HDG is a very efficient way to improve accuracy. In order to take advantage of the latter fact and move towards more efficient codes for computing wave propagation, as it has been postulated in previous works, it is natural to think about increasing the resolution at the wave front only. The option of doing this in a very convenient 


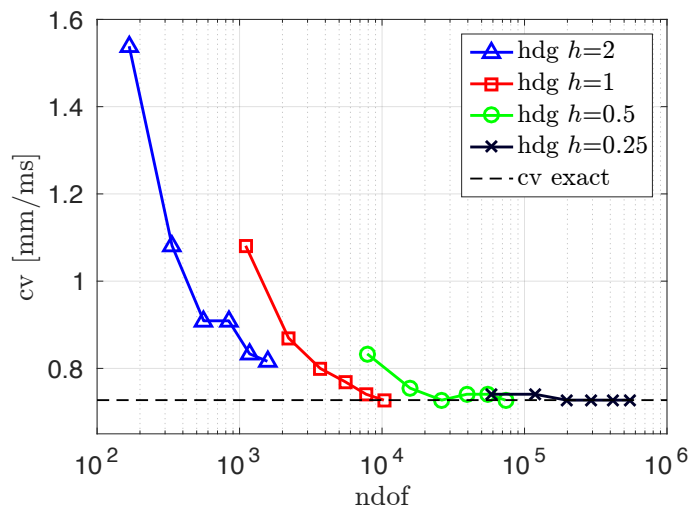

(a) number of degrees of freedom (ndof)

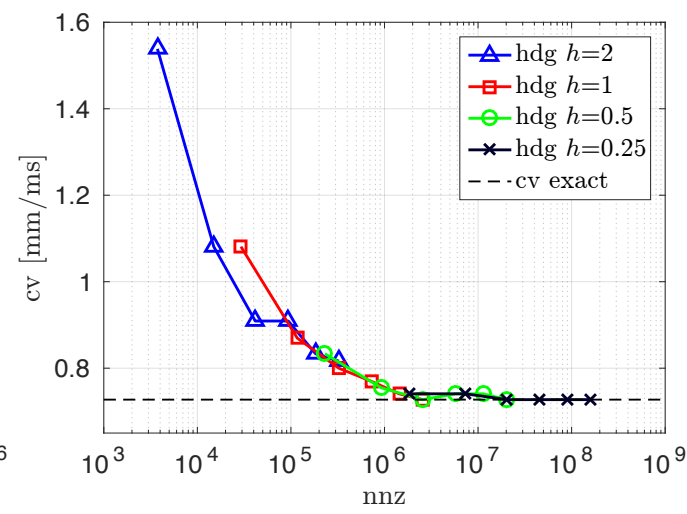

(b) number of non-zeros in system matrix (nnz)

Figure 8. This is a closeup of the results for HDG from Figures 7 and 7 . The conduction velocity for polynomial order $p=1,2,3,4,5$ and 6 is plotted.

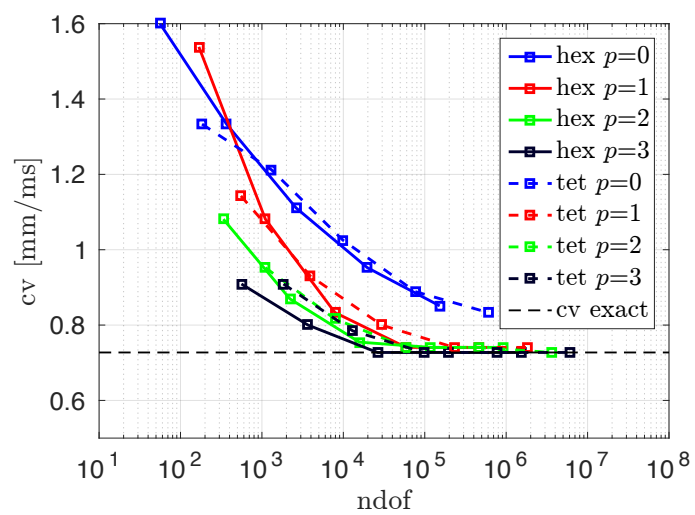

(a) number of degrees of freedom (ndof)

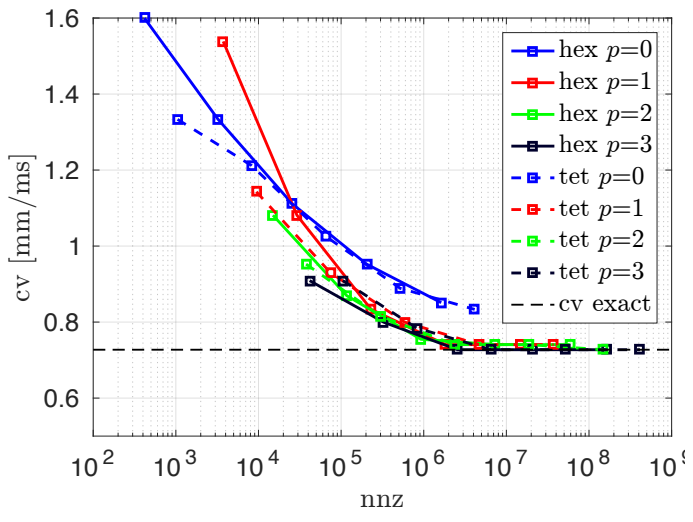

(b) number of non-zeros in system matrix (nnz)

Figure 9. Comparison between hexahedral (hex) and tetrahedral (tet) elements. The conduction velocity is plotted for element sizes of $h=2,1,0.5,0.25$ and $0.125 \mathrm{~mm}$. The difference between the element types is relatively small.

way is our main motivation for using HDG. Therefore, we now show the results of HDG using a p-adaptive approach.

We start the simulation with element order of $p=0$ everywhere, define a maximal polynomial order $p_{\max }$, and apply the adaptivity strategy specified in Section 2.6. Figure 10 shows the result of a p-adaptive simulation for element size $h=0.5 \mathrm{~mm}$ and $p_{\max }=10$. As expected, the method selects a high polynomial degree at the position of the steep electrochemical wave front, and after some time, when the wave has passed, the polynomial degree decreases again.

We present the results for different values of $h, p_{\max }$ and $e_{t o l}$ in more detail in Table II. by comparing the error between computed conduction velocity and the exact one. The table shows the error of the constant polynomial degree, $p=p_{\max }$ everywhere, and the adaptive polynomial degree until degree $p_{\max }$. Note that the adaptive simulations always utilize the highest polynomial degree. To see the influence of the error tolerance we calculated the error indicator without normalizing for three different element sizes $h=2,1$ and $0.5 \mathrm{~mm}$, i.e., we use $A_{\gamma}=1$ in Equation (14). The tested error tolerances are $e_{t o l}=10^{-2}, 10^{-4}$ and $10^{-8}$ (see Table II. From Table II we can see that the error decreases with increasing polynomial degree for the p-constant method a large tolerance. This 
is not the case for the p-adaptive method with an error tolerance of $e_{t o l}=10^{-2}$, i.e. p-adaptivity with a large error tolerance is not able to reproduce the p-constant solution. Only for small element sizes and small polynomial degrees the method is able to approximate the result of the p-constant method. Decreasing the error tolerance leads to a better approximation of the p-constant result as it can be seen in Table II for $e_{t o l}=10^{-4}$ and $e_{t o l}=10^{-8}$. However, a smaller error tolerance increases the number of high-order elements (Figure 11). For a coarse tolerance $\left(\right.$ e.g. $e_{t o l}=10^{-2}$ ) the number of elements with high order over time is small and it also decreases rapidly again. Decreasing the tolerance increases the number of high-order elements and over time the decrease of the number of high-order element is slower (Figure 11). A further decrease of the tolerance $e_{t o l}=10^{-8}$ results in a large number of high-order elements without returning to low order once the wave front has passed. The elements remain at high order until the action potential is returned to resting potential (Figure 11). This results almost in a constant polynomial degree over time. Thus, the smaller the error tolerance the more the constant method is approached.

Comparing different error tolerances for different element sizes shows large differences if the error indicator is not normalized (Figure 12). For an error tolerance of $e_{t o l}=10^{-4}$ the element order does not decrease in the simulation with element size $h=2 \mathrm{~mm}$. Using a normalized error indicator calculation as stated in Equation (14) and an error tolerance of $e_{t o l}=10^{-4}$ we can see that in all cases the polynomial degrees return to low order (Figure 12) and also the difference between constant and adaptive method is small (Table III). Figure 13 shows the distribution of the polynomial degrees at different times for a calculation with a normalized error indicator for a mesh with $h=0.5 \mathrm{~mm}$ element size. Slight differences in the polynomial order along the width of the geometry are due rounding errors. This asymmetry in the polynomial order distribution arises only after many time steps due to accumulated effects of an interger decision (which degree) from a continuous field that is almost the same (subject to roundoff). The polynomial degree increases when the electrochemical wave arrives and after around $10 \mathrm{~ms}$ the polynomial degree decreases again. Thus, our results verify that the error indicator calculation according to Equation (14) is a good option for localizing high degrees to the elements close to the wave front.

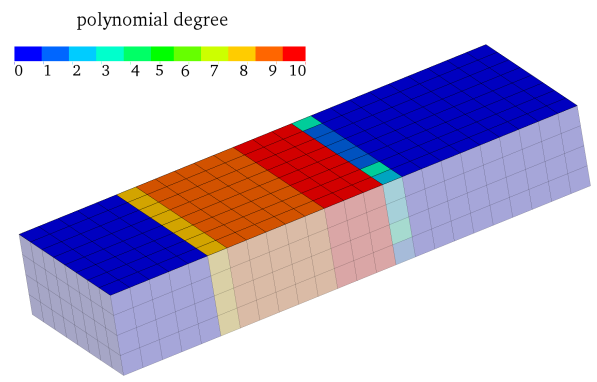

(a) polynomial degree distribution

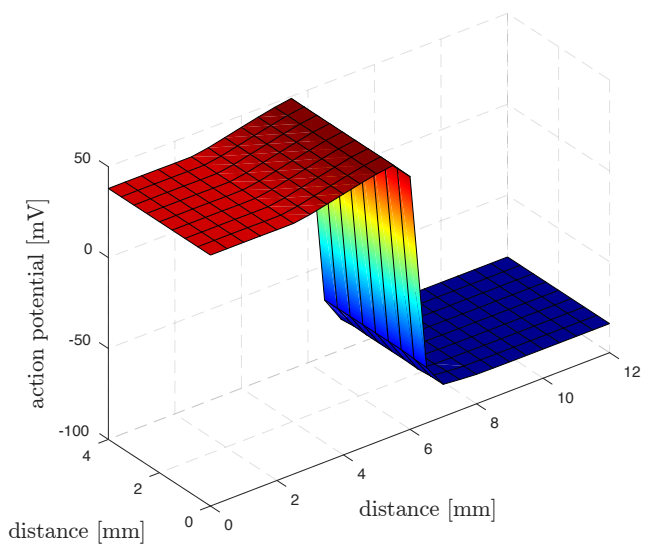

(b) action potential

Figure 10. Polynomial degree and action potential at time $6 \mathrm{~ms}$. The element size is $0.5 \mathrm{~mm}$ and the tolerance was chosen as $e_{t o l}=10^{-4}$. (a) Distribution of the adaptively chosen polynomial degree. (b) Action potential of the upper surface. At the steep electrochemical wave front the polynomial degree is high.

\subsection{Computations on a real biventricular geometry}

To show the applicability of our method to real cardiac electrophysiology simulations, we use our p-adaptive HDG implementation to solve the electrophysiological propagation problem for a real human ventricle geometry. For this purpose we segmented the left and right ventricle from magnetic resonance images acquired in a healthy 33 year old female volunteer, with a dual-phase 


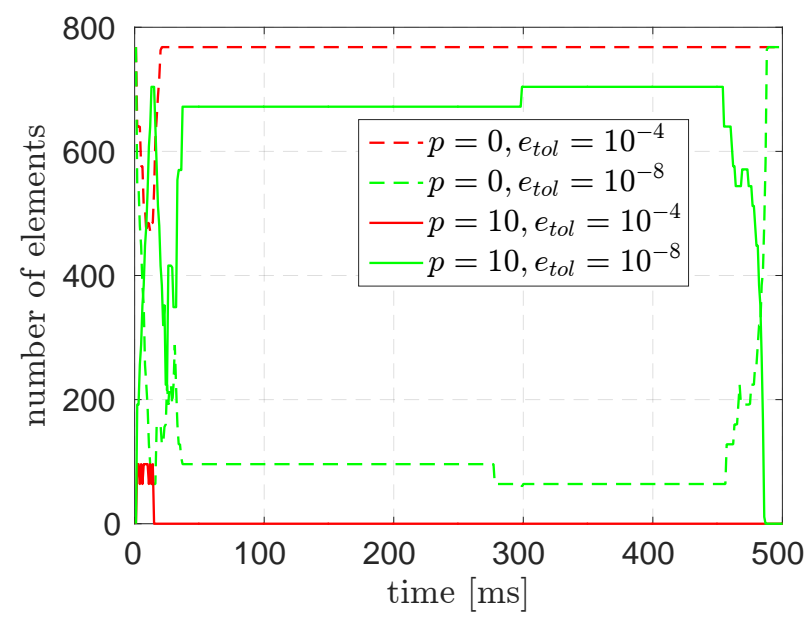

Figure 11. Number of elements with polynomial order of $p=0$ and $p=10$ during a p-adaptive simulation plotted over time. The element size is $h=0.5 \mathrm{~mm}$.

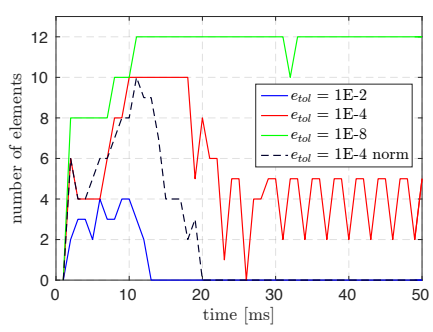

(a) $h=2 \mathrm{~mm}$

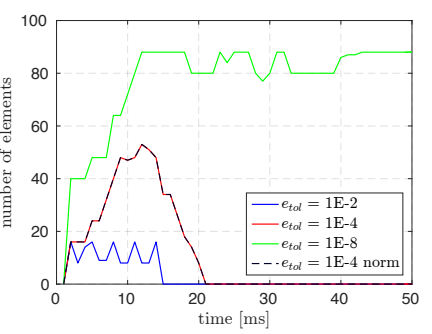

(b) $h=1 \mathrm{~mm}$

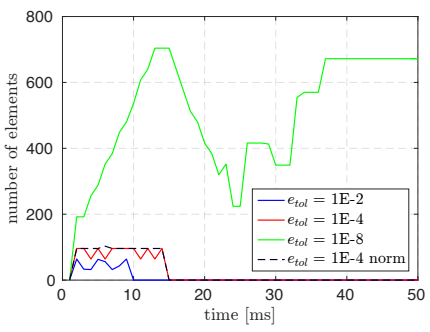

(c) $h=0.5 \mathrm{~mm}$

Figure 12. Number of elements with polynomial order of $p=10$ during a p-adaptive simulation plotted over time for different element sizes $h=2,1$ and $0.5 \mathrm{~mm}$ until a time of $50 \mathrm{~ms}$.

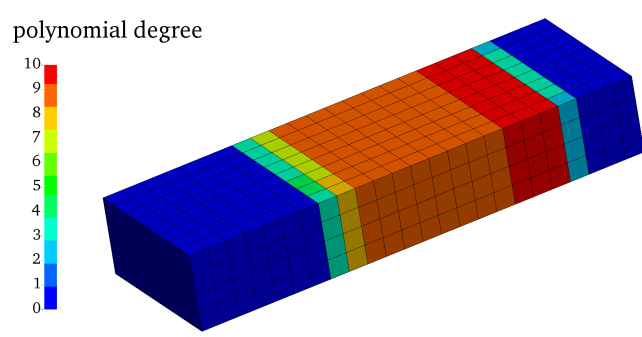

(a) time $10 \mathrm{~ms}$

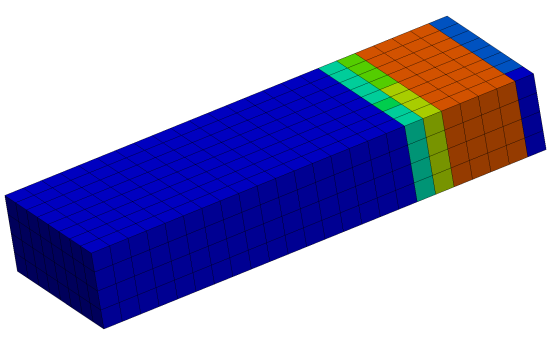

(b) time $17 \mathrm{~ms}$

Figure 13. Polynomial degree distribution for two different time steps for a mesh with element size of $0.5 \mathrm{~mm}$ and a normalized error indicator.

whole-heart 3D b-SSFP sequence [34] on a 1.5 T Philips Achieva MRI scanner, acquisition matrix $212 \times 209 \times 200$, acquired voxel size $2 \mathrm{~mm} \times 2 \mathrm{~mm} \times 2 \mathrm{~mm}$, repetition time $4.5 \mathrm{~ms}$, echo time $2.2 \mathrm{~ms}$, echo train length 26 and flip angle $90^{\circ}$. The diastasis was used to generate the computational mesh.

To investigate different levels of mesh refinement we use tetrahedral elements with a maximal element size of $2 \mathrm{~mm}, 1 \mathrm{~mm}$ and $0.5 \mathrm{~mm}$, which results in three meshes with 59801,393302 and 2904351 number of elements, respectively. On real heart geometries mesh refinements inevitably 


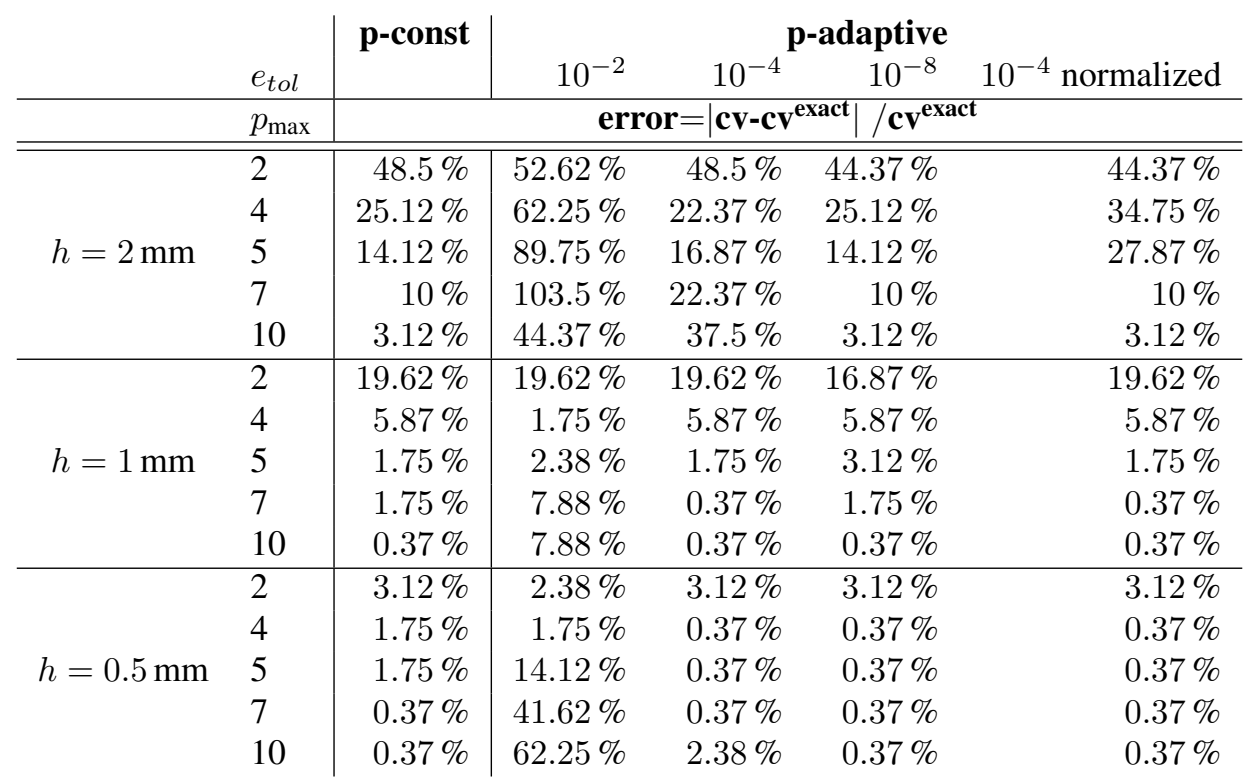

Table II. Comparison of the relative error to the exact conduction velocity of standard HDG method with the p-adaptive method for different polynomial degrees and different error tolerances $e_{t o l} \cdot p_{\max }$ is the maximal polynomial order allowed, $h$ is the element size and cv is the conduction velocity.

lead to slight changes of the geometry. Hence, we examine whether the difference in the geometry strongly influences the results, or coarser meshes with high-order elements are able to reproduce the activation propagation accurately.

We initiate the propagation of the electrical wave with a stimulus current on the apex. The maximal polynomial degree is set to five and at the beginning all polynomial degrees are set to zero. For computational reasons we use the integration rule defined in Equation (19) in this example for all polynomial degrees. The coarsest mesh shows a slightly faster activation than the finer ones, while only very small differences in the activation are visible between the two finer meshes (see Figure 14. The activation time of a sample point in the middle of the posterior wall at the junction between left and right ventricle has been captured for the different levels of refinement. The activation time of the sample point for the coarsest mesh is measured as about $40.7 \mathrm{~ms}$, for the finer one about $45.4 \mathrm{~ms}$ and for the finest mesh about $46.3 \mathrm{~ms}$.

When using non-adaptive HDG elements for the example with a maximal element size of $1 \mathrm{~mm}$ one would have to use 17739225 ndof during all time steps. In comparison, with the p-adaptive HDG method we have between 844725 minimal and 2275660 maximal ndof. The ndof averaged over the whole simulation depends on a particular case, of course, i.e. the geometry, the simulation time and the activation sequence influence the ndof over time. In our case, for an element size of $1 \mathrm{~mm}$ we show the average ndof until, e.g., the end of the activation or the end of the heart cycle. The averaged ndof can be seen in Table III. It decreases when simulating a larger part of the cardiac cycle, since the high polynomial degrees are used only at around the wave front. The decrease of the ndof is very useful, in particular when the calculated activation is coupled to mechanical simulation. In this case the activation takes place only in a short period of time, while the overall simulation is much longer.

The electrophysiological model can also be coupled to a mechanical model to calculate electromechanics in the heart. The mechanical simulation is solved using continuous finite elements, while for the electrophysiological simulation we use p-adaptive HDG discretization, but both meshes are the same. We couple the mechanical simulation through the action potential, which is stored for each element at the integration points. For more details about the coupling and the mechanical simulation we refer to our work described in [35]. In Figure 15 the activation time and 


\begin{tabular}{lc|c} 
specified simulation end & time & averaged ndof over time \\
\hline end of activation & $150 \mathrm{~ms}$ & 1709905 \\
end of systole & $300 \mathrm{~ms}$ & 1278179 \\
diastasis & $500 \mathrm{~ms}$ & 1107227 \\
end of heart cycle & $1000 \mathrm{~ms}$ & 988151
\end{tabular}

Table III. Ndof averaged from start until given time, for the example with $1 \mathrm{~mm}$ maximal element size.

the displacement at peak systole are shown for the ventricular geometry with a maximal element size of $1 \mathrm{~mm}$.

\section{CONCLUSION}

In this paper we proposed and analyzed the use of an adaptive high-order Hybridizable Discontinuous Galerkin (HDG) method for efficiently calculating the electric propagation in human hearts. As compared to classical DG methods an HDG discretization reduces the degrees of freedom through static condensation on the element level, so that only the degrees of freedom defined on the faces between the elements show up in the global system of equations. An advantage of the HDG method (as well as for other DG methods) is the simple usage of spatially varying highorder elements, due to the discontinuity between the elements that is captured through numerical fluxes. An approach with high-order elements in turn additionally needs a good approximation of the integral of the ionic current term. In this paper we have defined a rule for a suitable integration accuracy depending on the polynomial order and the cell model selected by the requirement to exactly integrate the leading current term. Furthermore we have defined and explained a practical choice of the stabilization parameter for the HDG discretization for the electrophysiological problem. Comparing CG and HDG methods, we have seen a similar performance for low-order elements, but an increase in efficiency for high-order elements.

The electrophysiology calculation is determined by a steep electrochemical wave front which travels through cardiac tissue. We proposed a simplification of an error indicator previously reported to localize it and to define an error indicator, which can be used to suggest the appropriate order of the element and in this way realize a p-adaptive HDG approach for cardiac electrophysiology. In summary, HDG shows great potential to efficiently solve large scale electrophysiological problems for complex geometries using coarse meshes and p-adaptive high-order elements, which can be additionally coupled with cardiac mechanical simulations.

\section{ACKNOWLEGEMENT}

The authors would like to thank Eva Sammut, King's College London, for her help with the image data used in this paper. Thanks also goes to Martina Weigl for her help in segmenting the biventricular geometry. 

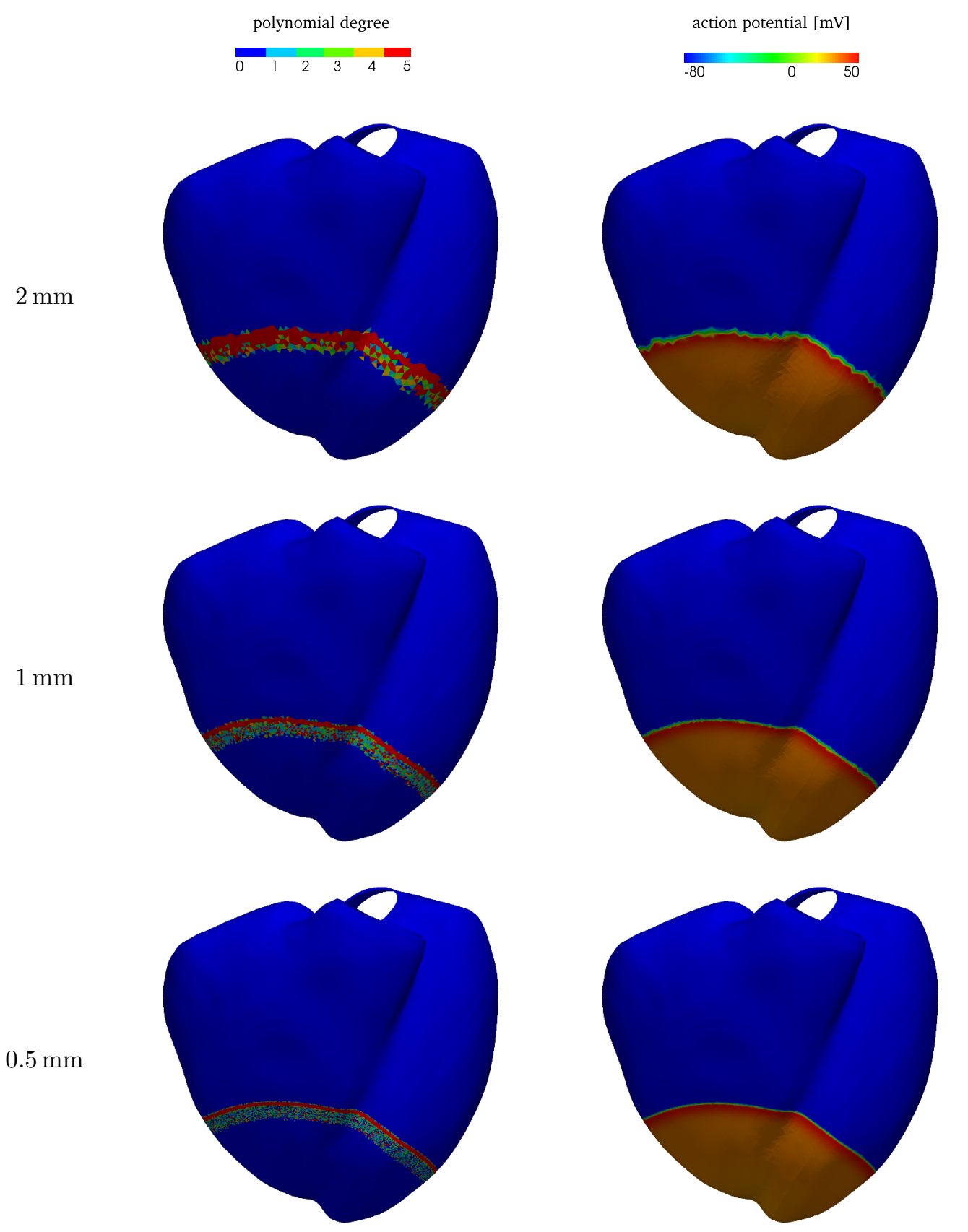

Figure 14. Polynomial degree and action potential at time $50 \mathrm{~ms}$.

\section{REFERENCES}

1. Chabiniok R, Wang VY, Hadjicharalambous M, et al.. Multiphysics and multiscale modelling, data-model fusion and integration of organ physiology in the clinic: Ventricular cardiac mechanics. Interface Focus 2016; 6(2), doi: 10.1098/rsfs.2015.0083.

2. Qu Z, Hu G, Garfinkel A, Weiss JN. Nonlinear and stochastic dynamics in the heart. Physics Reports 2014; doi: 10.1016/j.physrep.2014.05.002.

3. Niederer SA, Kerfoot E, Benson AP, et al.. Verification of cardiac tissue electrophysiology simulators using an Nversion benchmark. Philosophical Transactions of the Royal Society A: Mathematical, Physical and Engineering Sciences 2011; 369(1954):4331-4351, doi:10.1098/rsta.2011.0139. 


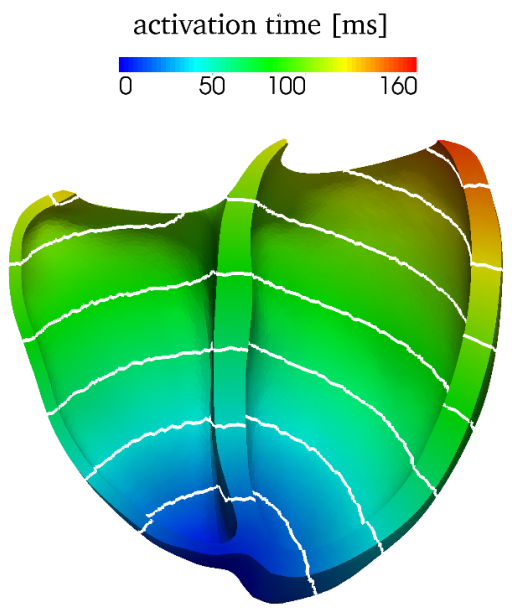

(a) Activation time

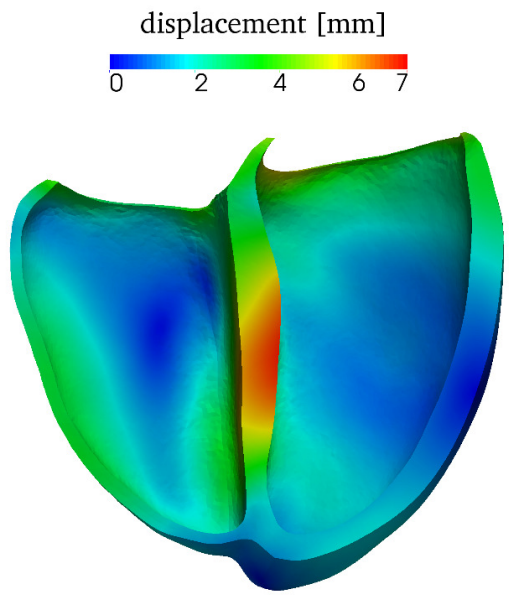

(b) Displacement

Figure 15. Activation time with isochrones at each $20 \mathrm{~ms}$ and the displacement at peak systole at about $300 \mathrm{~ms}$ from the activation onset for a real heart geometry with an element size of approximately $1 \mathrm{~mm}$.

4. Fischer G, Tilg B, Modre R, et al.. A Bidomain Model Based BEM-FEM Coupling Formulation for Anisotropic Cardiac Tissue. Annals of Biomedical Engineering 2000; 28(10):1229-1243, doi:10.1114/1.1318927.

5. Vigmond E, Aguel F, Trayanova N. Computational techniques for solving the bidomain equations in three dimensions. IEEE Transactions on Biomedical Engineering 2002; 49(11):1260-1269, doi:10.1109/TBME.2002. 804597.

6. Colli Franzone P, Pavarino LF. A parallel solver for reaction-diffusion systems in computational electrocardiology. Mathematical Models and Methods in Applied Sciences 2004; 14(06):883-911, doi:10.1142/S0218202504003489.

7. Göktepe S, Kuhl E. Computational modeling of cardiac electrophysiology: A novel finite element approach. International Journal for Numerical Methods in Engineering 2009; 79(2):156-178, doi:10.1002/nme.2571.

8. Linge S, Sundnes J, Hanslien M, Lines G, Tveito A. Numerical solution of the bidomain equations. Philosophical Transactions of the Royal Society A: Mathematical, Physical and Engineering Sciences 2009; 367(1895):19311950, doi:10.1098/rsta.2008.0306.

9. Pathmanathan P, Mirams GR, Southern J, Whiteley JP. The significant effect of the choice of ionic current integration method in cardiac electro-physiological simulations. International Journal for Numerical Methods in Biomedical Engineering 2011; 27(11):1751-1770, doi:10.1002/cnm.1438.

10. Krishnamoorthi S, Sarkar M, Klug WS. Numerical quadrature and operator splitting in finite element methods for cardiac electrophysiology. International Journal for Numerical Methods in Biomedical Engineering 2013; 29(11):1243-1266, doi:10.1002/cnm.2573.

11. Pezzuto S, Hake J, Sundnes J. Space-discretization error analysis and stabilization schemes for conduction velocity in cardiac electrophysiology. International Journal for Numerical Methods in Biomedical Engineering 2016; 32(10):n/a-n/a, doi:10.1002/cnm.2762.

12. Patelli AS, Dedè L, Lassila T, Bartezzaghi A, Quarteroni A. Isogeometric approximation of cardiac electrophysiology models on surfaces: An accuracy study with application to the human left atrium. Computer Methods in Applied Mechanics and Engineering 2017; 317:248-273, doi:10.1016/j.cma.2016.12.022.

13. Lines GT, Grottum P, Tveito A. Modeling the electrical activity of the heart: A Bidomain Model of the ventricles embedded in a torso. Computing and Visualization in Science 2002; 5(4):195-213, doi:10.1007/ s00791-003-0100-5.

14. Trangenstein JA, Kim C. Operator splitting and adaptive mesh refinement for the Luo-Rudy I model. Journal of Computational Physics 2004; 196(2):645-679, doi:10.1016/j.jcp.2003.11.014.

15. Belhamadia Y. A Time-Dependent Adaptive Remeshing for Electrical Waves of the Heart. IEEE Transactions on Biomedical Engineering 2008; 55(2):443-452, doi:10.1109/TBME.2007.905415.

16. Quan W, Evans SJ, Hastings HM. Efficient integration of a realistic two-dimensional cardiac tissue model by domain decomposition. IEEE Transactions on Biomedical Engineering 1998; 45(3):372-385.

17. Heidenreich E, Rodríguez J, Gaspar F, Doblaré M. Fourth-order compact schemes with adaptive time step for monodomain reaction-diffusion equations. Journal of Computational and Applied Mathematics 2008; 216(1):3955, doi:10.1016/j.cam.2007.04.011.

18. Cherry EM, Greenside HS, Henriquez CS. A space-time adaptive method for simulating complex cardiac dynamics. Physical Review Letters 2000; 84(6):1343.

19. Cherry EM, Greenside HS, Henriquez CS. Efficient simulation of three-dimensional anisotropic cardiac tissue using an adaptive mesh refinement method. Chaos: An Interdisciplinary Journal of Nonlinear Science 2003; 13(3):853865, doi:10.1063/1.1594685. 
20. Franzone PC, Deuflhard P, Erdmann B, Lang J, Pavarino LF. Adaptivity in Space and Time for ReactionDiffusion Systems in Electrocardiology. SIAM Journal on Scientific Computing 2006; 28(3):942-962, doi:10.1137/ 050634785 .

21. Whiteley JP. Physiology Driven Adaptivity for the Numerical Solution of the Bidomain Equations. Annals of Biomedical Engineering 2007; 35(9):1510-1520, doi:10.1007/s10439-007-9337-3.

22. Deuflhard P, Erdmann B, Roitzsch R, Lines GT. Adaptive finite element simulation of ventricular fibrillation dynamics. Computing and Visualization in Science 2009; 12(5):201-205, doi:10.1007/s00791-008-0088-y.

23. Arthurs CJ, Bishop MJ, Kay D. Efficient simulation of cardiac electrical propagation using high-order finite elements II: Adaptive p-version. Journal of Computational Physics 2013; 253:443-470, doi:10.1016/j.jcp.2013. 07.011.

24. Arthurs CJ, Bishop MJ, Kay D. Efficient simulation of cardiac electrical propagation using high order finite elements. Journal of Computational Physics 2012; 231(10):3946-3962, doi:10.1016/j.jcp.2012.01.037.

25. Sevilla R, Hassan O, Morgan K. An analysis of the performance of a high-order stabilised finite element method for simulating compressible flows. Computer Methods in Applied Mechanics and Engineering 2013; 253:15-27, doi:10.1016/j.cma.2012.09.001.

26. Giorgiani G, Fernández-Méndez S, Huerta A. Hybridizable discontinuous Galerkin p-adaptivity for wave propagation problems. International Journal for Numerical Methods in Fluids 2013; 72(12):1244-1262, doi: 10.1002/fld.3784.

27. Cockburn B, Gopalakrishnan J, Lazarov R. Unified Hybridization of Discontinuous Galerkin, Mixed, and Continuous Galerkin Methods for Second Order Elliptic Problems. SIAM Journal on Numerical Analysis 2009; 47(2):1319-1365, doi:10.1137/070706616.

28. Whiteley JP. An Efficient Numerical Technique for the Solution of the Monodomain and Bidomain Equations. IEEE Transactions on Biomedical Engineering 2006; 53(11):2139-2147, doi:10.1109/TBME.2006.879425.

29. Fernández MA, Zemzemi N. Decoupled time-marching schemes in computational cardiac electrophysiology and ECG numerical simulation. Mathematical Biosciences 2010; 226(1):58-75, doi:10.1016/j.mbs.2010.04.003.

30. Nguyen N, Peraire J, Cockburn B. An implicit high-order hybridizable discontinuous Galerkin method for linear convection-diffusion equations. Journal of Computational Physics 2009; 228(9):3232-3254, doi:10.1016/j.jcp. 2009.01.030.

31. Bueno-Orovio A, Cherry EM, Fenton FH. Minimal model for human ventricular action potentials in tissue. Journal of Theoretical Biology 2008; 253(3):544-560, doi:10.1016/j.jtbi.2008.03.029.

32. Kléber AG, Rudy Y. Basic Mechanisms of Cardiac Impulse Propagation and Associated Arrhythmias. Physiological Reviews 2004; 84(2):431-488, doi:10.1152/physrev.00025.2003.

33. Hesthaven JS, Warburton T. Nodal Discontinuous Galerkin Methods: Algorithms, Analysis, and Applications. No. 54 in Texts in applied mathematics, Springer: New York, 2008.

34. Uribe S, Tangchaoren T, Parish V, et al.. Volumetric Cardiac Quantification by Using 3D Dual-Phase Whole-Heart MR Imaging. Radiology 2008; 248(2):606-614, doi:10.1148/radiol.2482071568.

35. Hörmann JM, Bertoglio C, Nagler A, et al. Multiphysics Modeling of the Atrial Systole under Standard Ablation Strategies. Cardiovascular Engineering and Technology 2017; 8(2):205-218, doi:10.1007/s13239-017-0308-z. 